\title{
Adolescent prescription opioid misuse, illicit opioid use and overdose
}

\section{Michele K. Bohm, Heather Clayton}

National Center for Injury Prevention and Control, Centers for Disease Control and Prevention, Atlanta, Georgia, United State s

Objective

Given the evolving opioid overdose epidemic, we examined the interrelationships between nonmedical use of prescription opioids and illicit opioid use in adolescents.

\section{Introduction}

The number of overdose deaths involving illicit opioids such as heroin and illicitly-manufactured fentanyl (IMF) is now higher than deaths involving prescription opioids. Adolescents misusing prescription opioids are more likely to use heroin. Although nonmedical use of prescription opioids (NUPO) among

adolescents is decreasing, there is still relatively high prevalence of this behavior. Such high prevalence, along with the evolving epidemiology of the drug overdose epidemic as well as the association between NUPO and heroin use, signal that NUPO in adolescents is still an important issue. Understanding the interrelationships between NUPO and illicit opioid use in adolescents can inform prevention efforts. The purpose of this study is to: 1) present the magnitude of the drug overdose problem in adolescents, 2) compare the prevalence of heroin use and injection drug use (IDU) between students reporting NUPO and those not reporting NUPO, and 3) determine whether a dose-response relationship exists between these behaviors among adolescents. This information will be beneficial when focusing on adolescents at risk for heroin use by helping to determine whether any NUPO is associated with heroin use or if such risk is only noted at a higher frequency of NUPO behavior.

\section{Methods}

We analyzed data from two surveillance sources to capture adolescent overdose mortality and behavioral risk factors. Overdose death data for decedents aged 15 to 19 years were obtained for 2010 and 2016 from CDC WONDER, an online database with national mortality data based on death certificates for U.S. residents. We identified deaths involving prescription and illicit opioids using International Classification of Disease, 10th revision (ICD-10) codes for drug overdose deaths.

Each death is assigned one underlying cause of death code and the following identified overdoses: X40-44 (unintentional), X60-64 (intentional), X85 (homicide), or Y10-14 (undetermined intent). Additionally, for overdose deaths attributed to specific drugs or drug categories, ICD-10 multiple cause of death codes were used to determine the number of deaths involving any opioid, either prescription or illicit (T40.1-T40.4 and T40.6), prescription opioids (T40.2 or T40.3), heroin (T40.1), and heroin and/or synthetic opioids (e.g., fentanyl) excluding methadone (T40.1 or T40.4). We compared the proportion of overdose deaths involving prescription opioids that also involved heroin or synthetic opioids in 2010 and 2016 . The second data source, the 2017 national Youth Risk Behavior Survey (YRBS), a nationally representative cross-sectional survey of high school students, was analyzed to look at behavioral risk factors. We assessed lifetime NUPO (LNUPO) and calculated frequency of LNUPO by heroin use, injection drug use (IDU), and heroin/IDU using logistic regression models to g enerate adjusted prevalence ratios (aPR) and corresponding 95\% confidence intervals (CI). We used linear contrast analysis to determine dose-response relationships between frequency of LNUPO and heroin use, IDU and heroin/IDU.

\section{Results}

The number of adolescents aged 15 to 19 years who died of drug overdose increased from 831 in 2010 (3.8 per 100,000$)$ to 873 in 2016 (4.1 per 100,000). While the proportion of overdose deaths involving prescription opioids declined during this time period, the proportion involving heroin and/or synthetic opioids, such as fentanyl increased. In 2016, two-thirds of overdose deaths among decedents aged 15 to 19 years involved either a prescription or illicit opioid. The percent of deaths involving prescription opioids that also involved heroin and/or synthetic opioids, such as fentanyl increased from 5\% in 2010 to $25 \%$ in 2016 . Using the 2017 YRBS sample, we estimate that $14 \%$ of high school students nationwide have ever used prescription opioids nonmedically in their lifetime. Compared to students reporting no LNUPO, students reporting LNUPO were more likely to report heroin use (9.2\% vs. $0.4 \%)$, IDU (7.8\% vs. $0.4 \%)$, and heroin/ IDU (10.1\% vs. $0.7 \%)$. We observed

ISDS Annual Conference Proceedings 2019. This is an Open Access article distributed under the terms of the Creative Commons AttributionNoncommercial 3.0 Unported License (http://creativecommons.org/licenses/by-nc/3.0/), permitting all non-commercial use, distribution, and reproduction in any medium, provided the original work is properly cited. 
ISDS 2019 Conference Abstracts

a positive dose-response relationship with frequency of LNUPO. Adjusted prevalence ratios for heroin, IDU and heroin/IDU increased with increasing frequency of LNUPO and were even significantly higher among those reporting just one or two occasions of LNUPO than among those reporting no LNUPO.

\section{Conclusions}

Our findings on opioid-involved drug overdose mortality and opioid use patterns confirm NUPO is still a concern for adolescents. We report a five-fold increase, from 2010 to 2016, in the percent of adolescent overdose deaths involving prescription opioids that also involved illicit opioids such as heroin and/or IMF. This may reflect deliberate polysubstance use among adolescents using prescription opioids nonmedically, but should also be considered in the context of stable prevalence of reported heroin use in YRBS and the National Survey on Drug Use and Health. In addition to issues with selfreport bias, adolescents may not self- identify as a person who uses heroin, for example, if they unknowingly use counterfeit prescription pills that contain heroin or IMF. Health risk behaviors established in adolescence often continue into young adulthood and understanding associations between opioid initiation, misuse, and overdose is critical for prevention efforts. Although we found a dose-response relationship between the frequency of LNUPO and the prevalence of heroin and IDU, we also report significantly higher heroin use and IDU among students reporting just one or two occasions of LNUPO compared to students reporting no LNUPO. This underscores the importance of prevention efforts aimed at all adolescents who use prescription opioids nonmedically, with particular emphasis on those frequently misusing them. Clinical, community, and school-based efforts can address NUPO, noting these associations.

ISDS Annual Conference Proceedings 2019. This is an Open Access article distributed under the terms of the Creative Commons AttributionNoncommercial 3.0 Unported License (http://creativecommons.org/licenses/by-nc/3.0/), permitting all non-commercial use, distribution, and reproduction in any medium, provided the original work is properly cited. 